\title{
Obtención de hidrógeno utilizando la energía residual de las pilas usadas
}

\author{
José Antonio Guevara García ${ }^{\mathrm{a} *}$, Beatriz Morales Chamorro ${ }^{\mathrm{a}}$, Brian Manuel González Contreras ${ }^{\mathrm{b}}$, \\ Miguel Angel Munive Rojas ${ }^{\mathrm{c}}$
}

${ }^{a}$ Laboratorio de Investigación en Bioinorgánica y Biotecnología (LIByB). Fac. de Ciencias Básicas. Universidad Autónoma de Tlaxcala, Campus Apizaco, P.O. Box 140, 90300 . Apizaco, Tlaxcala. México.

${ }^{b}$ Maestría en Computación. Fac. de Ciencias Básicas, Ingeniería y Tecnología. Universidad Autónoma de Tlaxcala, Campus Apizaco, P.O. Box 140, 90300. Tlaxcala. México.

'P.E.de Ingeniería Mecánica. Fac. de Ciencias Básicas, Ingeniería y Tecnología. Universidad Autónoma de Tlaxcala, Campus Apizaco, P.O. Box 140, 90300. Tlaxcala. México .

*E-mail: jaguevarag@gmail.com

\section{Recibido 11 de septiembre de 2013, Aceptado 30 de noviembre de2013}

\section{Resumen}

Las pilas y baterías usadas causan cada vez más contaminación ambiental en México, debido al crecimiento de la demanda de equipo electrónico portátil que genera miles de toneladas de estos residuos al año. En México no se hace reciclado de pilas usadas porque es inviable económicamente una planta industrial para este propósito. El objetivo de este trabajo es la obtención de hidrógeno por electrólisis de agua utilizando la energía residual de las pilas usadas, como parte inicial de un proceso de reciclado. Las pilas fueron clasificadas por tipo y por marca, y separadas por carga. Se dispusieron en una batería de 24 pilas "AA" para alimentar a un electrolizador en forma de "U" para producir $\mathrm{H}_{2}$ y $\mathrm{O}_{2}$. Los datos de corriente y voltaje, así como el volumen de $\mathrm{H}_{2}$, se determinaron en función del tiempo, para cuantificar la producción de corriente promedio por pila $(\mathrm{mAh})$ y el rendimiento de $\mathrm{H}_{2}$. Los resultados mostraron un rendimiento de producción de $\mathrm{H}_{2}$ de $2.35 \%$, y una producción de corriente promedio por pila de $1321 \mathrm{mAh}$, que es más del $50 \%$ del reportado por el fabricante para una pila alcalina nueva. Una planta de reciclado de pilas usadas de 80 toneladas mensuales, producirían, con una estimación a la baja, $4961 \mathrm{kWh}$ solamente utilizando las pilas "AA" de más de $1.0 \mathrm{~V}$ (cerca del 90\%). Este trabajo contribuye a hacer atractivo económicamente el reciclado de pilas y a diseñar planes de manejo sustentable de la energía y de los materiales contenidos en estos residuos.

Palabras clave: pilas, reciclado, hidrógeno, electrólisis, manejo sustentable.

\section{Introducción}

La contaminación ambiental producida por la eliminación de las pilas y baterías usadas es una de las principales preocupaciones en México debido al rápido crecimiento de la demanda de equipo electrónico portátil que utiliza a estas como fuente de energía y que, ante la inexistencia de reciclado, genera miles de toneladas de residuos peligrosos al año [1].

Uno de los argumentos que han evitado el reciclado de pilas usadas en México es el balance económico negativo que se tendría en una planta industrial para este fin. Nuevas consideraciones que revierten esta situación son: el incremento en su producción y consumo; la aparición de nuevas tecnologías que incorporan metales con mejor cotización como $\mathrm{Cd}$ y Li; la demanda de materia prima de los propios productores de pilas como el Dióxido de Manganeso Electrolítico (DME) y el Hidróxido de Litio (HL), sobrepasará en pocos años la producción y las reservas, y como consecuencia se incrementa su precio considerablemente.

En un trabajo anterior [2], nuestro grupo de investigación determinó el voltaje residual de 700 pilas de tipo "AA" seleccionadas al azar de las cuatro marcas de mayor incidencia después de una campaña de recolección de pilas usadas. Sus resultados mostraron que el $36 \%$ de las pilas tuvieron un voltaje residual en el intervalo de 1.2-1.4 V, mientras que $70 \%$ de las pilas tuvo voltajes mayores de $1.0 \mathrm{~V}$, observándose desperdicio de energía en estos dispositivos. Un cálculo aproximado señala que en México se comercializan todos los años alrededor de 420 millones de pilas solo de tipo "AA" (NMX-AA-104-SCFI-2006), de las cuales 189 millones contendrían la energía residual suficiente disponible para otras aplicaciones. La recuperación de la energía residual de las pilas y baterías usadas no se ha tenido en cuenta hasta ahora [3], y podía ser económicamente atractivo.

En este trabajo se reporta una opción de aprovechamiento de la energía residual de las pilas usadas por electrólisis de agua y obtención de hidrógeno. La electrólisis del agua es una tecnología muy importante para una producción de hidrógeno a gran escala. El hidrógeno es la energía que se espera que sea útil como vector energético principal en un futuro cercano [4], para la generación de electricidad vía las pilas de combustible de hidrógeno $(\mathrm{PCH})$. Este proceso se propone como parte inicial del reciclado, lo que aunado a la recuperación de metales y producción de materia prima (DME y HL), puede conducir al manejo sustentable de este recurso, y ayudar a evitar que se agoten las reservas de metales como Mn y Li. Además, este proceso de reciclado integral impulsaría la creación de empresas y la generación de fuentes de trabajo, y evitaría el impacto 
ambiental de los metales tóxicos en los rellenos.

\section{Parte experimental}

\subsection{Recolección de pilas usadas}

Se recabaron un total de 7876 pilas que se clasificaron por tipo, resultando que 6020 (76\%) fueron "AA"; de estas $28 \%$ correspondieron a marcas ilegales. Se tomó una muestra de 3625 pilas "AA" para medir su voltaje, obteniéndose la gráfica de distribución de voltajes por medio del programa Origin 8. Posteriormente, se seleccionaron 5 conjuntos de 24 pilas "AA" usadas de varias marcas con valores de voltaje residual en el intervalo $1.4-1.6 \mathrm{~V}$ para utilizarse en las baterías.

\subsection{Producción de hidrógeno con pilas usadas}

Se construyeron varios paneles (baterías) de pilas usadas tipo "AA", para proporcionar la corriente y voltajes necesarios para alimentar el electrolizador de manera continua. Cada panel consiste en un bloque de unicel con 14 horadaciones, con 2 pilas en cada horadación. Las pilas se colocan alternando las terminales $(+)$ y $(-)$, y cada 8 pilas se conectan en serie, sumando sus voltajes, y luego las tres series de 8 pilas se conectan en paralelo, sumando sus corrientes (ver Figura 1). Las conexiones se hacen con alambres de cobre y, para asegurar el contacto, la batería se coloca entre las tapas de una prensa que se atornilla con la presión mínimamente suficiente.

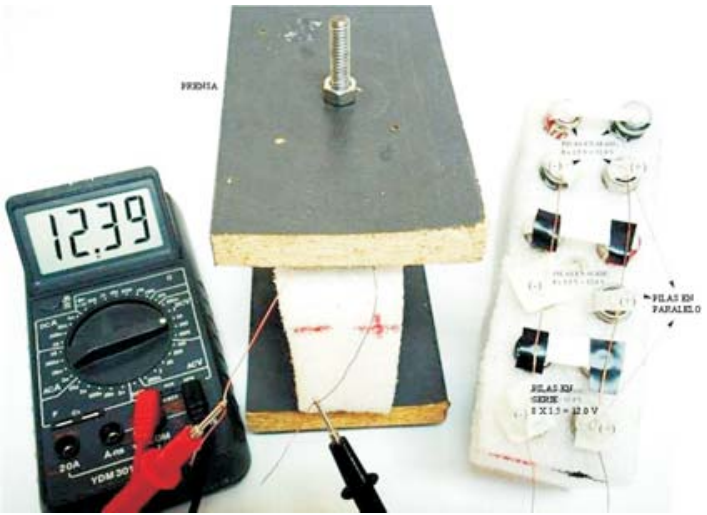

Figura 1. A la derecha, batería de 24 pilas usadas "AA" conectadas en serie (cada 8) y en paralelo. Al centro, la batería calzada en una prensa para asegurar los contactos. A la izquierda, multímetro mostrando la lectura de voltaje de la batería.

Se construyó un electrolizador para la producción de hidrógeno por electrólisis. Este consiste de un tubo en "U" con salidas para gases, electrodos de acero inoxidable en láminas acopladas a los tapones. El electrolizador contiene una solución de hidróxido de sodio al $30 \%$.

Posteriormente se acoplaron el panel (batería) y el electrolizador para las pruebas de producción de hidrógeno. La terminal negativa de la batería se conectó en serie a un amperímetro, para determinar la corriente. El voltaje se determinó utilizando un multímetro conectado en paralelo a la batería. El volumen de hidrógeno se midió por el desplazamiento del émbolo de una jeringa de vidrio conectada a la salida del tubo en "U". El volumen producido de hidrógeno se midió cada 10 segundos. Además, se anotó la temperatura del experimento en cada momento y la presión atmosférica, para normalizar el volumen de hidrógeno.

Con este equipo se realizaron ensayos de electrólisis (ver Figura 2). De cada corrida se obtuvieron las gráficas de volumen de producción de hidrógeno contra tiempo y las gráficas de voltaje y corriente contra tiempo. De estas últimas gráficas, se determinó la producción de corriente (mAh). El consumo de energía (Wh), se calculó como el área bajo las curvas de voltaje contra corriente.

Para calcular la producción de corriente promedio por pila, se promediaron los valores de mAh de tres gráficas correspondientes a otros tantos ensayos individuales, y este promedio se dividió entre 24 pilas de que consta cada batería.

\section{Resultados y discusión}

Como puede verse en la Figura 3, la mayor parte de las pilas usadas "AA" tiene un voltaje en el rango 1.4-1.6 $\mathrm{V}$, y la distribución de frecuencias no sigue una distribución normal.

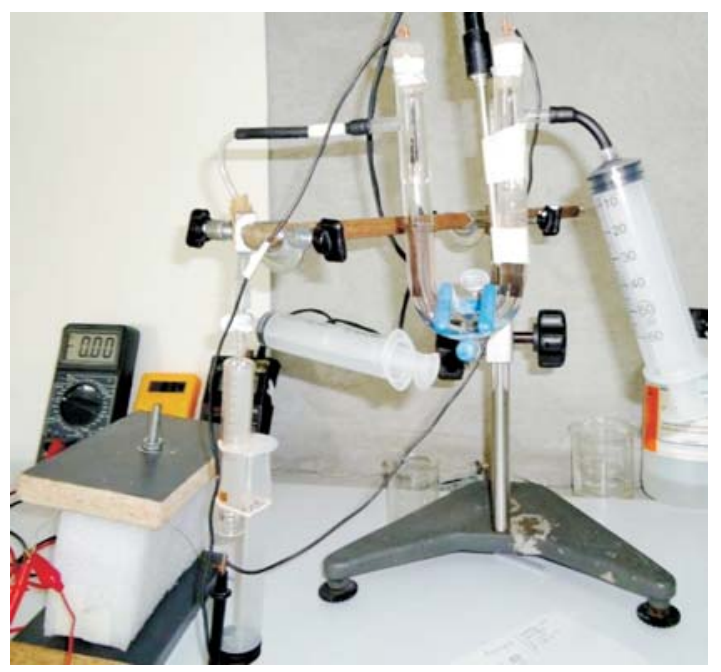

Figura 2. Imagen del equipo del electrolizador en " $U$ " alimentado por la batería de pilas, y accesorios para la determinación de volumen, voltaje, corriente y temperatura.

Una posible explicación es que las pilas se utilizan en dispositivos de alta demanda de corriente en la mayoría de los casos. Inicialmente, la reacción de descarga se lleva a cabo en las zonas exteriores de los electrodos, donde el transporte de masa es más rápido. Sin embargo, conforme se mantiene la demanda, las reacciones se llevan a cabo dentro de la estructura de los electrodos, produciendo un sobrepotencial por difusión, dando lugar a una disminución en la cinética de transferencia de electrones y a una polarización por concentración [5]. Es común que el usuario cambie las pilas tan pronto como dejan de proporcionar suficiente poder para operar el dispositivo y que no las re-utilice en equipos menos demandantes de energía, lo cual podría tomar ventaja de la energía residual. 


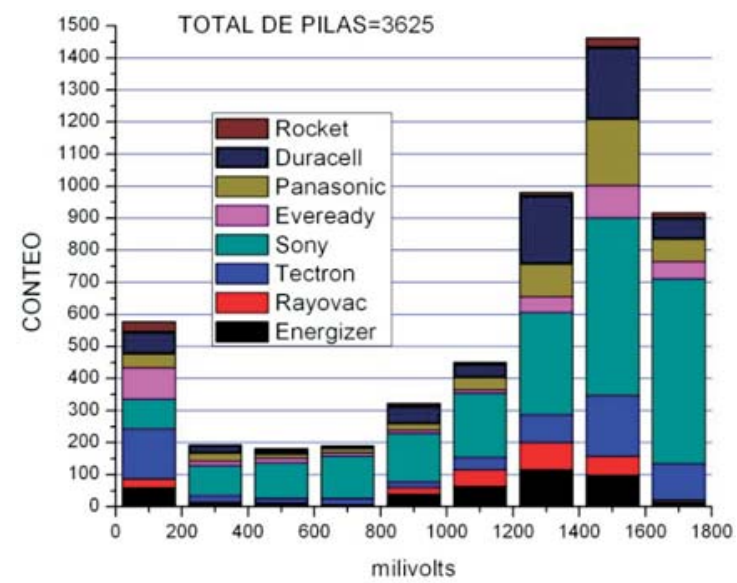

Figura 3. Distribución de voltaje residual de pilas usadas tipo "AA" por marcas (colores) y en total.

El rendimiento de producción de hidrógeno fue de $2.35 \%$, mientras que en la literatura se reportan rendimiento máximos del $12 \%$, utilizando equipos especializados [6]. La producción de corriente promedio por pila fue de $1321 \mathrm{mAh}$. Esto representa alrededor del $50 \%$ del reportado para una pila alcalina nueva [7]. En la Figura 4 se reproduce una de las gráficas obtenidas de corriente vs voltaje y su ajuste por mínimos cuadrados (Origin 8.0). Los puntos experimentales observan varias inflexiones debido a que la batería no se utilizó de manera continua hasta su descarga, sino que se le dejó reposar por varios momentos para permitir su recuperación.

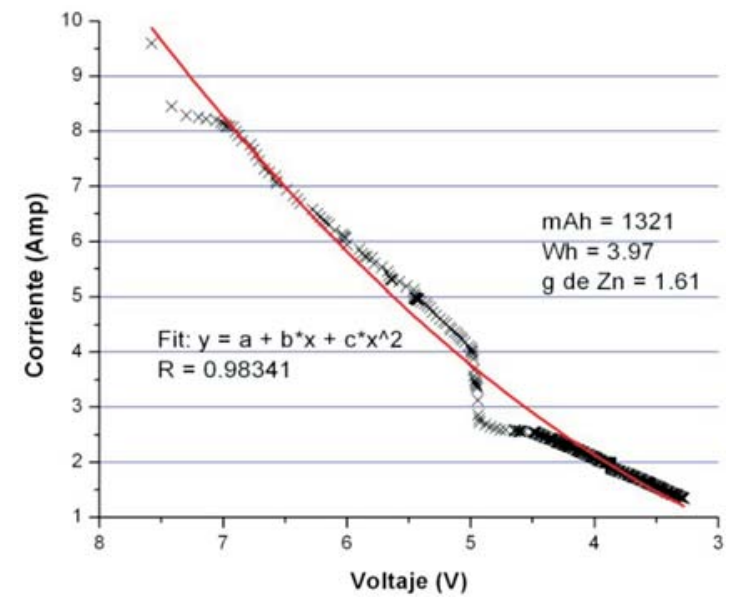

Figura 4. Gráfica resultante de corriente contra voltaje, con la ecuación de ajuste obtenida y la producción de corriente (mAh) y energía (Wh) promedio por pila.

Considerando una planta de reciclado de pilas usadas de 80 toneladas mensuales, se producirían, con una estimación a la baja, $4961 \mathrm{kWh}$ solamente utilizando las pilas "AA" de más de $1.0 \mathrm{~V}$ (cerca del 90\%, según Figura 3). Esto significa $1.5 \times 106$ mol-electrón disponibles para producción vía electroquímica. Si con esto se produce hidrógeno, entonces se obtendrían $1.8 \times 106 \mathrm{~L}$, suponiendo un rendimiento del 10\%. Esta energía puede ser utilizada también para producir parte del DME a partir del reciclado de pilas usadas, que en total ascendería a 103 ton de DME.

\section{Conclusiones}

Un porcentaje considerable de las pilas usadas (cerca del $90 \%$ de las pilas "AA") tienen una energía utilizable considerable (alrededor del 50\%). Esta energía puede ser recuperada y aprovechada en la misma forma que se utilizan paneles fotovoltaicos y constituir un ahorro de energía importante en una planta de reciclado. En la aplicación específica de producción de hidrógeno vía electrolítica, el rendimiento es bajo pero es susceptible de optimizarse hasta en un $12 \%$. Esto hace atractivo económicamente el reciclado de pilas y contribuye a diseñar un manejo sustentable de la energía y de los materiales contenidos en estos residuos.

\section{Referencias}

1. Montiel Corona, V.; Guevara García, J.A.; Reyes López, J.A.; Landry, T.D. Situación Actual de las Pilas Usadas en México. En Residuos Sólidos en Iberoamérica, Primera Edición; G.C. Díaz Trujillo, J.F. Gallardo Lancho, Eds.; Red Iberoamericana de Física y Química: Salamanca, España, 2012; pp. 150-169. ISBN 978-84-937437-5-8. www.sifyqa.org.es.

2. Guevara-García, J.A.; Montiel-Corona, V. J. Environmental Management. 2012, 95, S154-S157.

3. Sayilgan, E.; Kukrer, T.; Civelekoglu, G.; Ferella, F.; Akcil, A.; Veglio, F.; Kitis, M. Hidrometallurgy. 2009, 97, 158166.

4. Montiel Corona, V.; Sánchez Camarillo, A.R.; Guevara García, J.A. Revista CiBIyT. 2009, 3 (11), 35-42.

5. Hamann, C.H.; Hamnett, A.; Vielstich, W. Electrochemistry. Second ed.; Wiley-VCH Verlag $\mathrm{GmbH} \&$ Co. KGaA: Weinheim, 2007; pp. 444-447.

6. Gibson, T.L.; Kelly, N.A. Int. J. Hydrogen Ener. 2008, 33, 5931-5940.

7. Wiaux J.-P.; WaeflerJ.-P. J. Power Sources. 1995, 57, 6165. 